\title{
Deep learning techniques for bone surface delineation in ultrasound
}

\section{Conference Paper}

\section{Author(s):}

Ciganovic, Matija; Ozdemir, Firat (Di); Farshad, Mazda; Goksel, Orcun (i)

Publication date:

2019

Permanent link:

https://doi.org/10.3929/ethz-b-000350278

\section{Rights / license:}

In Copyright - Non-Commercial Use Permitted

Originally published in:

Proceedings of SPIE 10955, https://doi.org/10.1117/12.2512997 


\title{
Deep Learning Techniques for Bone Surface Delineation in Ultrasound
}

\author{
Matija Ciganovic $^{1,2}$, Firat Ozdemir ${ }^{1}$, Mazda Farshad ${ }^{2}$, Orcun Goksel ${ }^{1}$ \\ ${ }^{1}$ Computer-assisted Applications in Medicine, ETH Zurich, Switzerland; \\ ${ }^{2}$ Department of Orthopaedics, Balgrist University Hospital, University of Zurich, Switzerland
}

\begin{abstract}
For computer-assisted interventions in orthopedic surgery, automatic bone surface delineation can be of great value. For instance, given such a method, an automatically extracted bone surface from intraoperative imaging modalities can be registered to the bone surfaces from preoperative images, allowing for enhanced visualization and/or surgical guidance. Ultrasound (US) is ideal for imaging bone surfaces intraoperatively, being real-time, non-ionizing, and cost-effective. However, due to its low signal-to-noise ratio and imaging artifacts, extracting bone surfaces automatically from such images remains challenging.

In this work, we examine the suitability of deep learning for automatic bone surface extraction from US. Given 1800 manually annotated US frames, we examine the performance of two popular neural networks used for segmentation. Furthermore, we investigate the effect of different preprocessing methods used for manual annotations in training on the final segmentation quality, and demonstrate excellent qualitative and quantitative segmentation results.
\end{abstract}

\section{INTRODUCTION}

Bone surface segmentation from ultrasound (US) is an area of active research for computer-assisted interventions. For instance, a bone surface as extracted from intra-operative images can be used for registration to preoperative images 114 in order to achieve surgical guidance and navigation. US is particularly suited for this purpose, as it is real-time, affordable, and does not involve ionizing radiation. However, it inherently suffers from low signal-to-noise ratio (SNR), which renders a fully automated analysis of US frames challenging.

Many algorithms have been proposed for bone surface segmentation. For instance, Hacihaliloglu et al ${ }^{5}$ have proposed a method based on phase symmetry, $\underset{6}{6}$ exploiting the fact that bone surfaces typically appear as bright ridges in US images. Phase symmetry was also used in a later work ${ }^{3}$ in tandem with the gradient energy tensor.$^{7}$ Karamalis et al.$^{8}$ used a different characteristic feature of bone surfaces in US, in that they aim to detect the shadow regions of bone surfaces, namely the low-intensity regions in US images caused by the fact that bone surfaces reflect most of US energy, thereby occluding everything on the far side with respect to the US transducer. Combining Hacihaliloglu ${ }^{\sqrt{5}}$ and Karamalis ${ }^{8}$ along with attenuation features, Quader et al ${ }^{9}$ have introduced confidence-weighted local phase features (CPS). More recently, machine learning techniques have been used. Ozdemir et al ${ }^{10}$ detect bone surfaces by modelling US propagation in tissue using a factor graph and decision trees. Baka et al!11 first used random forests and logistic classifiers, and later deep learning ${ }^{12}$ based on U-Net ${ }^{13}$ Salehi et al ${ }^{1]}$ have recently proposed a state-of-the-art deep learning-based method for bone surface segmentation in US, by integrating the segmentation output of U-Net and a connected component analysis with the method from Jain et al ${ }^{14}$ for choosing the bone surface position in each vertical scanline as the middle pixel between the maximum intensity and the maximum gradient location.

In this work, we investigate the suitability of deep learning approaches for US bone surface delineation in an end-to-end fashion, i.e. no preprocessing of the input images and as little postprocessing of the network outputs as necessary to obtain single-pixel thick segmentation masks. In addition, we investigate the effect of various preprocessing methods of the manual labels on the training success, and thereby on the final segmentation quality. To enable comparison with inter-rater performance as an upper bound, all US frames from the test set were labelled by two independent annotators. 


\section{METHODS}

Architectures. We investigate deep networks based on two popular convolutional neural network (CNN) architectures for image segmentation: U-Net $\frac{13}{13}$ and Fully Convolutional Neural Netwoks (FCN) $\frac{15}{{ }^{15}}$ specifically, the FCN-8s. However, in contrast to using the models as proposed originally, we use $(i)$ batch normalization $1 \frac{16}{1}$ at each convolutional layer, which is known to greatly stabilize training, and (ii) only half the number of channels at all layers compared to the originally proposed models, which decreased the model complexity significantly.

In order to be able to handle arbitrarily sized inputs, deep-learning based approaches typically process images by subdividing them into equal-sized patches, which are then processed individually. We herein use a relatively large patch size, since in order to characterize hyperechoic responses correctly as bone surfaces in US, a spatially large anatomical context is needed. For instance, similarly to bones, tendons may also appear as bright ridges in US. However, while a characteristic shadow obstructs visibility beneath bone surfaces in US, tissue behind tendons remains visible. Thus, in order to capture such shadowing regions as early as possible in the network, we chose the patch size to be relatively large while still fitting the deep models and a sufficiently large batch size in GPU memory. Patch-wise predictions are then fused using a logical OR to the original image size.

Preprocessing annotations. For annotation, bone surfaces (used for training and evaluation of the networks) are typically delineated as single-pixel thick ("hairline") contours at the bone surface responses in US. The main issue arising from this type of data is that only very few image pixels get labelled as bone surface in every image (in our case, only around $0.6 \%$ of all image pixels in the training set were labelled). Such a level of class imbalance often has a severe impact on the training success. To remedy this, we propose to use weighted cross-entropy as the loss function, where the weight for each label is computed as the inverse ratio of the corresponding pixels in the training set (i.e, the weight for the bone label, which has an occurrence rate of $0.6 \%$ of the image pixels in the training set, is set to be 0.994 ). Hypothesizing that such hairline annotations may severely degrade training performance, we investigate several preprocessed versions of these manual annotations:

1. Hairline: We used the original manually-annotated hairline curve as is (see Figure $1 \mathrm{~b}$ ).

2. Dilated: Since bone surfaces appear in US images as bright ridges of several mm depth, $\frac{14}{14}$ even expert annotations are prone to localization errors due to ambiguity in the exact location of the bone surface within the ridge. Hence, network predictions which do not precisely overlap with the manual annotations should not be considered completely wrong by default. Using a dilated gold standard label, in combination with a weighted cross entropy loss function, we ensure the predictions close enough to the gold standard are not penalized (see Figure 1c). Such an approach was also used in Baka et al. ${ }^{12}$ The number of pixels for dilation was chosen based on US pulse length and typical surface appearance/thickness, such that the resulting gold standard stripe would capture the entire bone surface response in the US images.

3. Blurred We also used an isotropically Gaussian-blurred version of the hairline annotations, with the motivation similar to that of the dilated gold standard described above. However, with blurring, the penalty of a pixel labelled as 'bone' increases exponentially as the predictions move away from the original labels (see Figure 1d). Blurring kernel size, i.e. standard deviation $\sigma$, was chosen again based on the visual thickness of average bone surfaces empirically as above.

4. Shadow: One defining aspect of the appearance of bone surfaces in US is a shadowing region beneath such surfaces $\frac{10}{10}$ i.e. areas that are hypoechoic due to the high reflective properties of bone surfaces in addition to high attenuation of US in bone. Accordingly, in each scanline in the gold standard, we mark all pixels below bone surface annotations with an additional shadow label, thereby explicitly drawing the attention of the network to such shadowing beneath bone surfaces.

Because one of the main motivations for automatized bone surface delineation from US is registration, we are interested in obtaining a single-pixel thick segmentation output. Thus, we postprocess the segmentation outputs from the networks by selecting, for each connected component separately, only the pixel at the middle row of each non-zero scanline vertically. 


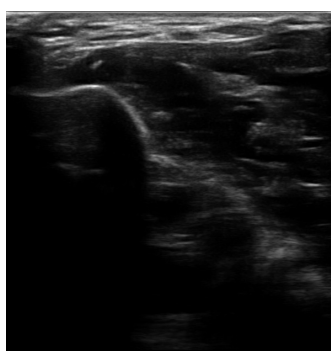

(a)

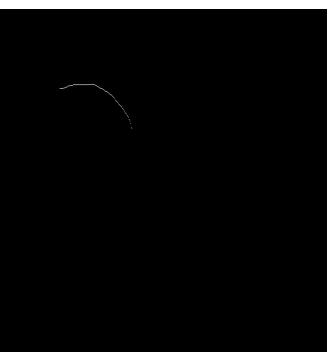

(b)

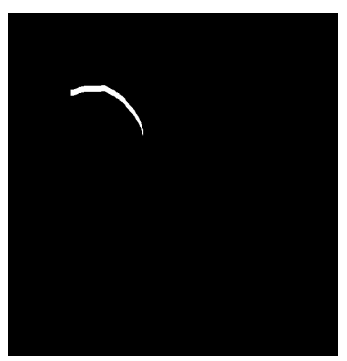

(c)

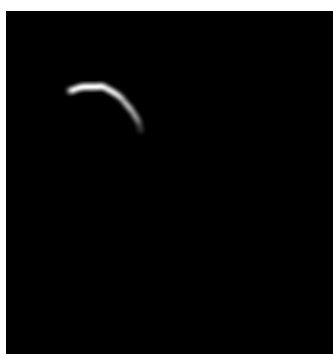

(d)

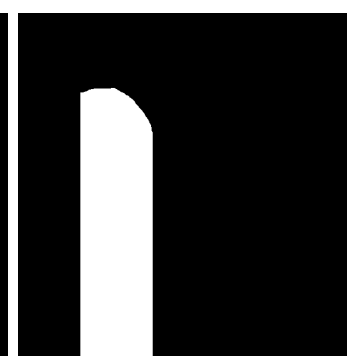

(e)

Figure 1: US frame and different versions of manual annotations used as gold standard. (a) Example US frame used for training; (b) original hairline manual annotation; (c) annotation after morphological dilation using a line-shaped structuring element of 10 pixels length; (d) annotation after Gaussian blurring with zero mean and $\sigma=5$ pixels; (e) annotation extended with shadowing label. Note that in (e), the bone contour itself and the shadow region below have different labels.

\section{EXPERIMENTS AND RESULTS}

Data. We have acquired a total of 1800 labelled in-vivo US-images of the left forearm from two volunteers, both from the dorsal and volar aspects. The data was collected within the context of an earlier work, 2$]$ and bone surfaces were only labelled where they were clearly visible and distinguishable from other tissue. This was done using the Stradwin* software. For acquisitions, an L14-5 linear-array US transducer was used with Sonix Touch and Tablet machines (Ultrasonix Medical Corporation, Richmond, BC, Canada). Imaging depths varied between 40 and $50 \mathrm{~mm}$, at an imaging frequency of 6.66 or $10 \mathrm{MHz}$. With these differing parameters, we aimed to cover a range of imaging settings and thereby take into account images with various intensity distributions for testing the generalization of the neural networks.

Training. For dilating the manual labels, a line-shaped, vertical structuring element of 10 pixels in length was used. For blurring, we used a zero-mean Gaussian with $\sigma=5$ pixels. As a large patch size, we chose $448 \times 448$ pixels. Accordingly, all US images were divided into 4 overlapping patches of $448 \times 448$ pixels. Of the 1800 collected frames, two thirds (approximately 1400 images, first volunteer) have been used for training, of which around 160 images have been used as the validation set during training. The remaining 400 images (second volunteer) were used for testing. Thus, the models were only exposed to US frames of one of the volunteers during training, and were evaluated exclusively on US images of the second one. Each compared model was trained for 20000 iterations, which took approximately 24 hours on an NVidia Titan XP GPU. We have used the Adam ${ }^{17}$ optimizer for training, with $\beta_{1}=0.9, \beta_{2}=0.999, \alpha=0.001$, as proposed by Kingma et al. During training, the batch size was set to 5 patches. All networks were implemented using the TensorFlow library ${ }^{\dagger}$

Evaluation. We compare the CNN architectures described in Section 2 with our implementation of a recent deep-learning based approach ${ }^{1}$ as the baseline. This baseline method performs a largest component analysis of the neural network output. Since our dataset consists of US images of the forearm, at most two bones are visible in one image patch at the same time (radius and ulna). As a consequence, we chose to keep the two largest components of the neural network output when running the baseline network.

During test time, we set the batch size of our models to 60 for FCN (corresponding to 15 full images) and 40 for U-Net and the baseline (corresponding to 10 full images). For evaluation, we used root-mean squared error RMSE (using distances between the gold standard labels and their nearest prediction label), which quantifies an overall agreement between gold standard annotations and the corresponding segmentation maps. We also computed the one-way Hausdorff (oHD) distance between gold standard labels and their nearest prediction label, as well as the symmetric Hausdorff (sHD) distance. Note, however, that we compute those only for scanlines that contain both, a gold standard- and a prediction label. Doing otherwise would punish bone surfaces that may be correctly detected by our algorithm, but that are not labelled by the human annotator. Finally, we use a metric

\footnotetext{
*http://mi.eng.cam.ac.uk/ rwp/stradwin/

thttps://www.tensorflow.org/
} 
Table 1: Quantitative segmentation results on the test set, given as as mean \pm std $[\mathrm{mm}]$. We report the root mean squared error (RMSE), one-way Hausdorff (oHD), symmetric Hausdorff (sHD) and scanline mean error (SME). RMSE, oHD and SME are computed using distances from gold standard annotations to nearest prediction label. SME, oHD and sHD are computed only for scanlines that contain both, a gold standard- and a prediction label.

\begin{tabular}{|c|c|c|c|c|}
\hline Method (annotation) & RMSE & SME & oHD & sHD \\
\hline baseline [Salehi et al! $\left.^{[}\right]$ & $0.34 \pm 1.03$ & $0.22 \pm 0.26$ & $0.81 \pm 2.03$ & $1.43 \pm 3.69$ \\
\hline U-Net (hairline) & $\mathbf{0 . 1 8} \pm \mathbf{0 . 4 5}$ & $0.14 \pm 0.08$ & $\mathbf{0 . 4 6} \pm \mathbf{1 . 0 7}$ & $\mathbf{1 . 1 5} \pm \mathbf{2 . 2 4}$ \\
\hline U-Net (dilated) & $0.23 \pm 0.67$ & $0.14 \pm 0.07$ & $0.61 \pm 1.55$ & $1.21 \pm 2.72$ \\
\hline U-Net (blurred) & $0.86 \pm 2.24$ & $0.14 \pm 0.08$ & $2.41 \pm 4.39$ & $2.72 \pm 4.99$ \\
\hline U-Net (shadow) & $0.25 \pm 0.8$ & $0.14 \pm 0.09$ & $0.65 \pm 1.77$ & $1.24 \pm 2.92$ \\
\hline \hline FCN (hairline) & $0.65 \pm 1.94$ & $0.13 \pm 0.05$ & $1.52 \pm 3.95$ & $2.46 \pm 5.07$ \\
\hline FCN (dilated) & $0.24 \pm 0.8$ & $0.13 \pm 0.08$ & $0.68 \pm 1.99$ & $1.63 \pm 3.25$ \\
\hline FCN (blurred) & $0.95 \pm 2.3$ & $\mathbf{0 . 1 2} \pm \mathbf{0 . 0 6}$ & $2.75 \pm 4.46$ & $2.86 \pm 4.63$ \\
\hline FCN (shadow) & $0.67 \pm 2$ & $\mathbf{0 . 1 2} \pm \mathbf{0 . 0 5}$ & $1.63 \pm 4.07$ & $1.92 \pm 4.76$ \\
\hline \hline Inter-Annotator & $0.91 \pm 2.76$ & $0.13 \pm 0.06$ & $2.57 \pm 5.77$ & $2.69 \pm 6.07$ \\
\hline
\end{tabular}

called the scanline mean error SME: For each US scanline that contains a gold standard label and a prediction label, we measure the distance between the ground truth pixel and the nearest predicted pixel. The average stored distance over all such scanlines is then the SME. This evaluation metric was inspired by the fact that bone surface responses in US typically exhibit a thickness of several $\mathrm{mm}$, so even if two segmentation algorithms correctly respond to only the bone surface responses, they may still differ as to where exactly along the vertical scanlines they detect the single-pixel bone surface. We acquired manual annotations of two independent annotators for all images in the test set, which we used to quantify the inter-annotator disagreement using the same evaluation metrics described above. This allows us to compare the neural network segmentation performance to human annotator agreement.

Results. Quantitative results are shown in Table 1. Methods compared are reported together with the manual annotation type used during training in parentheses. U-Net(hairline) shows the best performance for almost all evaluation metrics. Figure 2 shows qualitative examples using U-Net(hairline) (best performing model), and Figure 5 shows the qualitative evaluation of the baseline method. As can be seen in Figure 2 , even in the worst-case segmentations of the respective error metrics, segmentation quality is still quite high.

For U-Net, FCN, and the baseline, the segmentation inference time for a full batch (15 full frames for FCN, 10 for U-Net and baseline) is $390 \pm 5 \mathrm{~ms}$ on average, while the postprocessing (thinning) takes $20 \mathrm{~ms}$ per image on average.

\section{DISCUSSION}

The quantitative results shown in Table 1 suggest that U-Net(hairline) provides the best segmentation performance, as it has the lowest error scores in almost all considered error metrics. However, it is important not to succumb to certain pitfalls that these numbers create. Recall that, in all error metrics (except for sHD), the distances considered to compute them were from the gold standard labels to their nearest prediction label. Measuring the distances vice versa, the evaluation process would represent algorithms that outperform human segmentation as inferior, since any bone surface response that was wrongfully ignored by the manual annotator but correctly identified as such by the algorithm would increase the computed error. However, in our case, we have found that algorithms exhibiting lower error scores do so at the cost of selectivity. Thus, while they respond to most of the surfaces that were also labelled by the annotator, they generate more false positives than algorithms with a higher error. This can be seen by comparing Figures 2 and 4 , which show qualitative results of U-Net(hairline) and FCN (blurred), i.e., the configurations with lowest- and highest error values, respectively. For additional visualisation, Figures 3 and 5 show qualitative results of FCN(hairline) and the baseline method, respectively. Thus, we conclude that performing manual label pre-processing for training favors specificity over sensitivity, making the networks more selective for bone surface responses, particularly in the case of Gaus- 


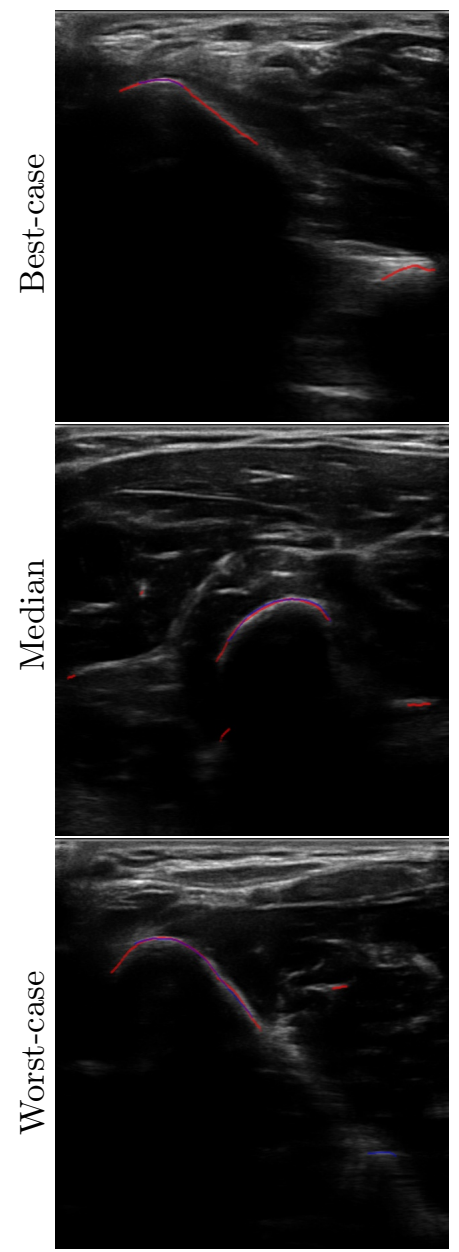

(a) RMSE

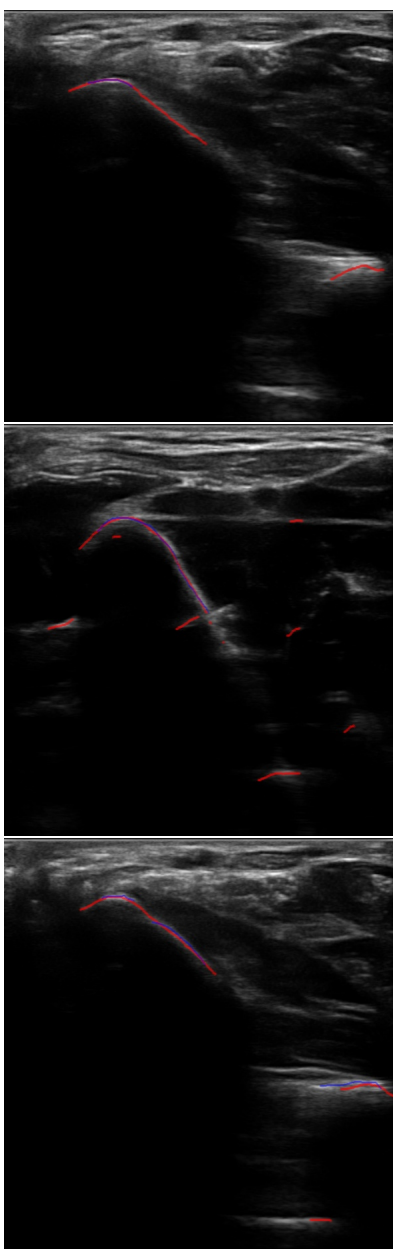

(b) SME

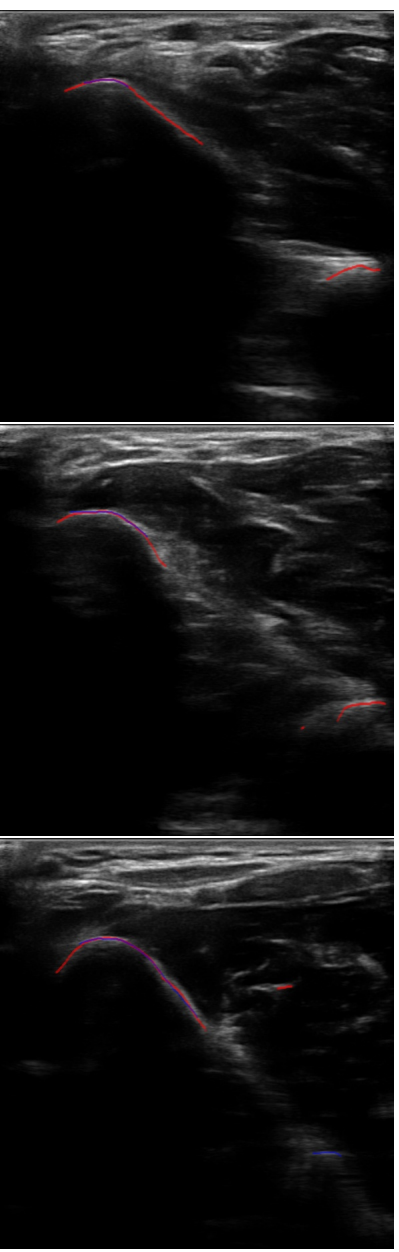

(c) oHD

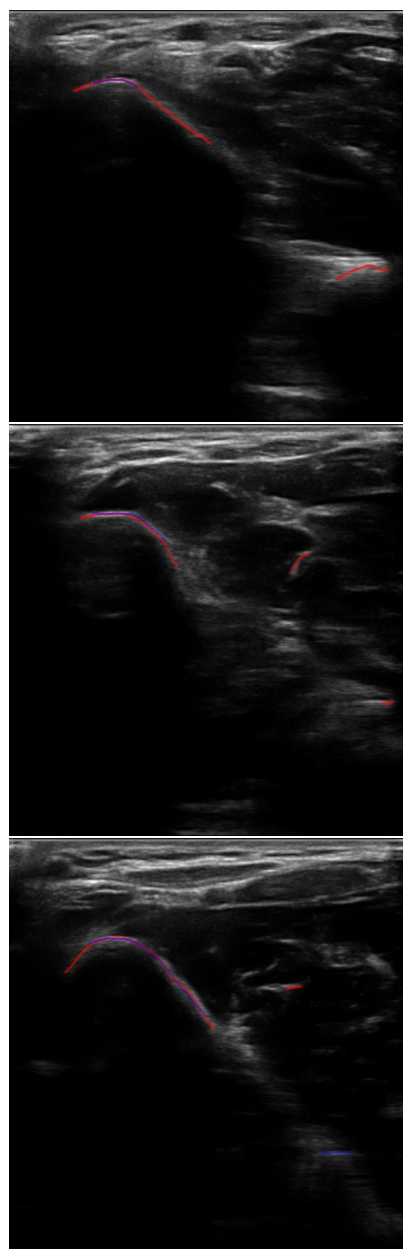

(d) $\mathrm{sHD}$

Figure 2: Qualitative evaluation of U-Net(hairline), which exhibited lowest errors. Red points show segmentations as performed by the network, blue points show the manual gold standard annotation. The top row shows images with minimum error (best-case) for the corresponding column evaluation metric, the middle row shows medianerror cases, and the bottom row shows the images for the maximum error (worst-case) for each corresponding metric. Note the relatively high number of noise in e.g. the median case of the SME.

sian blurring of the manual labels. Overall, FCN exhibits higher error values than the corresponding U-Net configuration. This might be due to the simpler up-sampling path as compared to the U-Net.

In general, our experiments suggest that simple CNNs are suitable to give excellent bone surface delineations from US. Comparing the network performances to the inter-annotator disagreement, we conclude that the network performances are close to that of a human annotator. As for the baseline method, one can conclude from Figure 5 that its main drawback is the use of largest component analysis that is applied on the U-Net output. While we have found that such a form of response 'pruning' was necessary to reduce the number of false positives (which we speculate to result from the fact that no batch normalization was used), in order to successfully choose the correct components, one would need to know the number of bony structures in the imaging field-of-view in advance. Choosing this preset number too small would lead to the algorithm discarding true bone surface responses, and choosing it too large may cause an increase in false positive responses. 


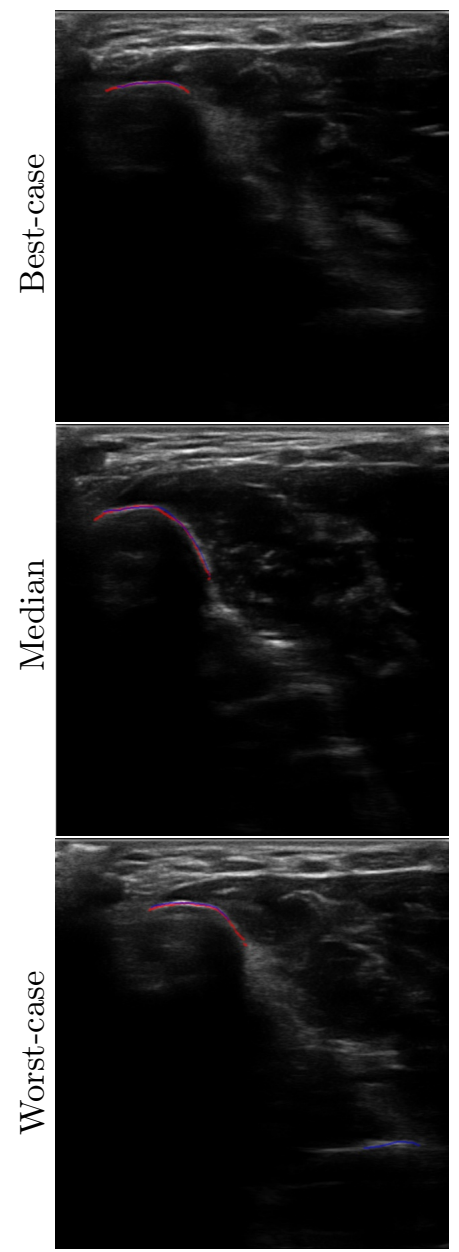

(a) RMSE

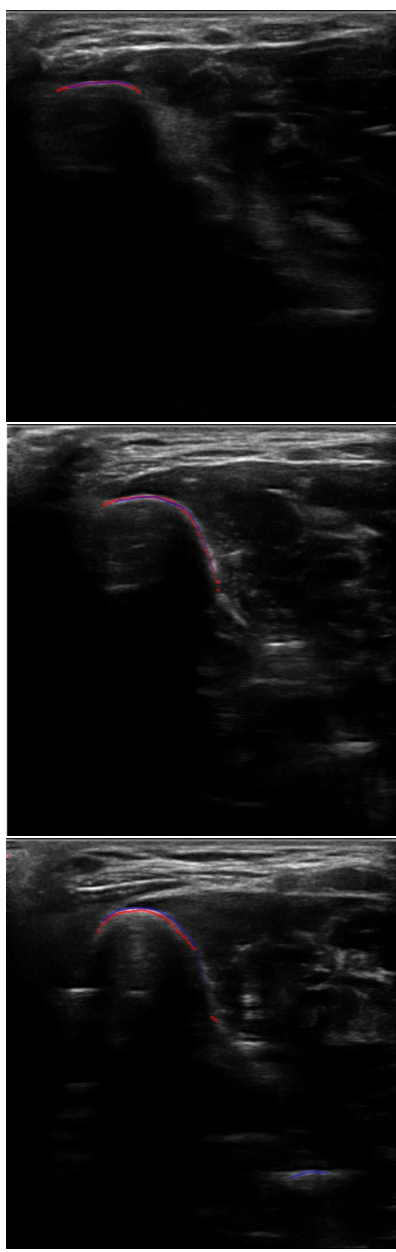

(b) SME

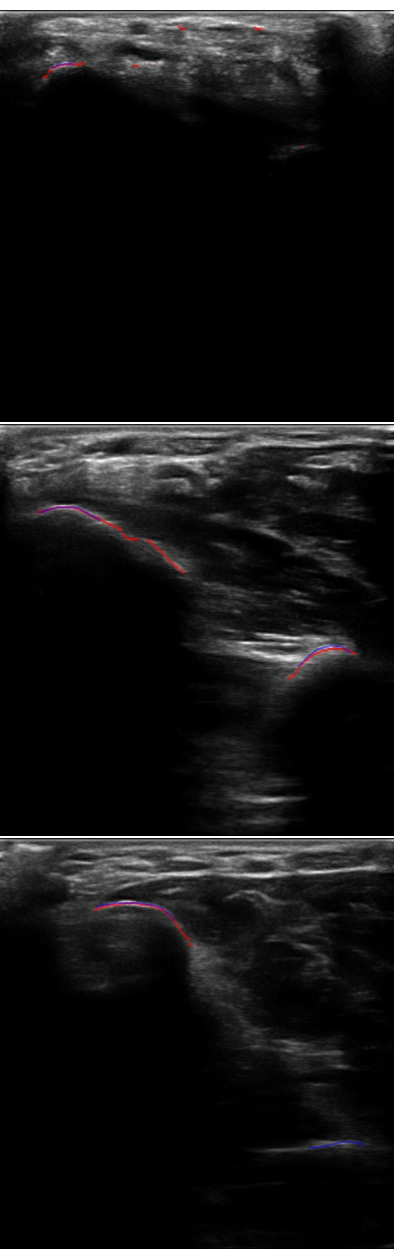

(c) oHD

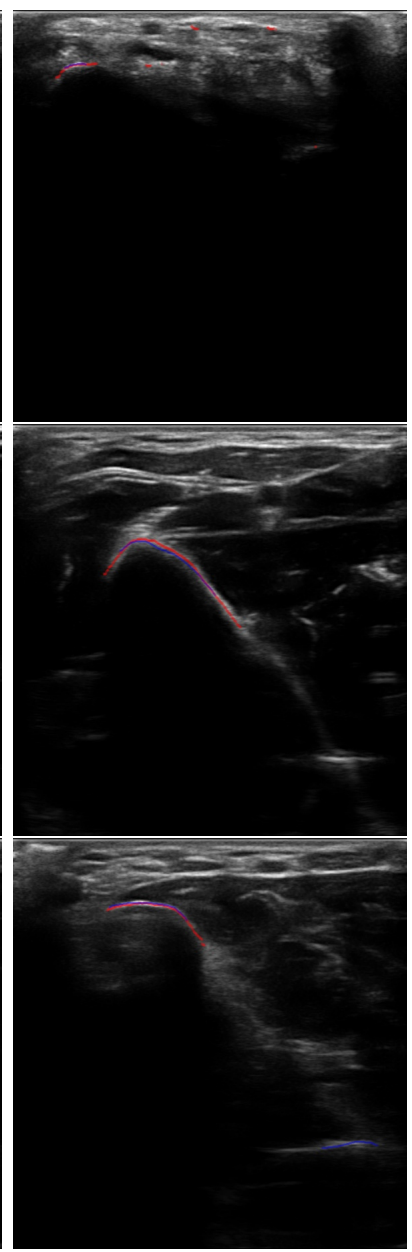

(d) $\mathrm{sHD}$

Figure 3: Qualitative evaluation of our proposed FCN(hairline) method. Red points show segmentations as performed by the network, blue points show the manual gold standard annotation. The top row shows images with minimum error (best-case) for the corresponding column evaluation metric, the middle row shows medianerror cases, and the bottom row shows the images for the maximum error (worst-case) for each corresponding metric. Network segmentations look better than those generated with U-Net(hairline) as shown in Figure 2 despite the higher quantitative error.

\section{CONCLUSIONS AND FUTURE WORK}

We have investigated deep learning approaches to delineate bone surfaces in US images. To this end, we have compared CNNs based on two popular network architectures, U-Net and FCN, along with various ways of annotation preprocessing for training. We used a dataset consisting or 1800 manually annotated US images of the forearm from two volunteers. We found that applying pre-processing to the manual labels help reducing false positives, increasing their specificity in their respective segmentations. Thus, while quantitatively the best performing model architecture for bone surface segmentation was found to be U-Net with batch normalization at every convolutional layer trained on single-pixel manual annotations, qualitatively we have found that network configurations with higher quantitative errors produced cleaner segmentations, in particular when Gaussian blurring of the labels was used. This might be beneficial for applications such as registration. A comparably poor performance of the baseline method (U-Net + postprocessing as proposed by Salehi et al., 1 cf. Table 1) is likely due to the lack of batch normalization, which has been shown in numerous applications to improve neural network training. Another potential drawback of the baseline is that the largest connected component 


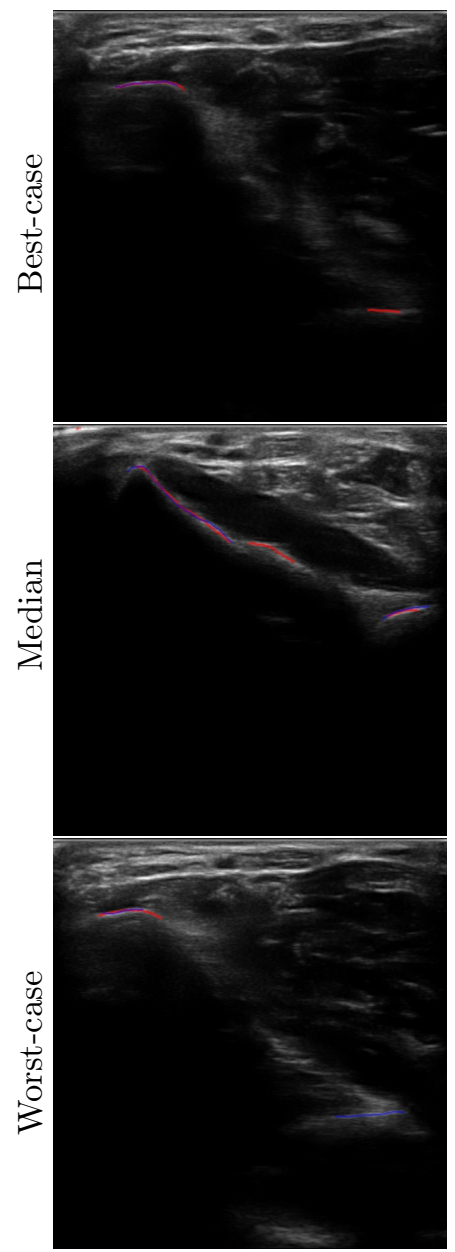

(a) RMSE

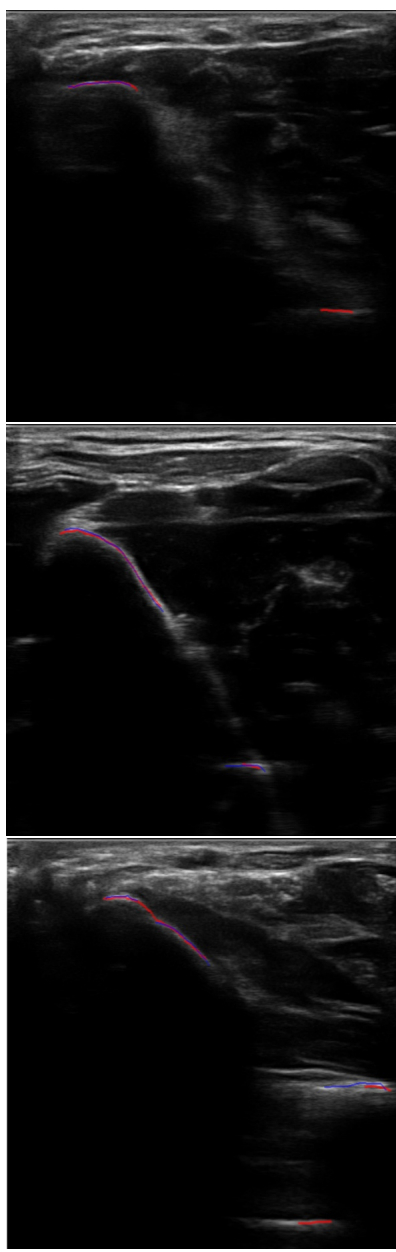

(b) SME

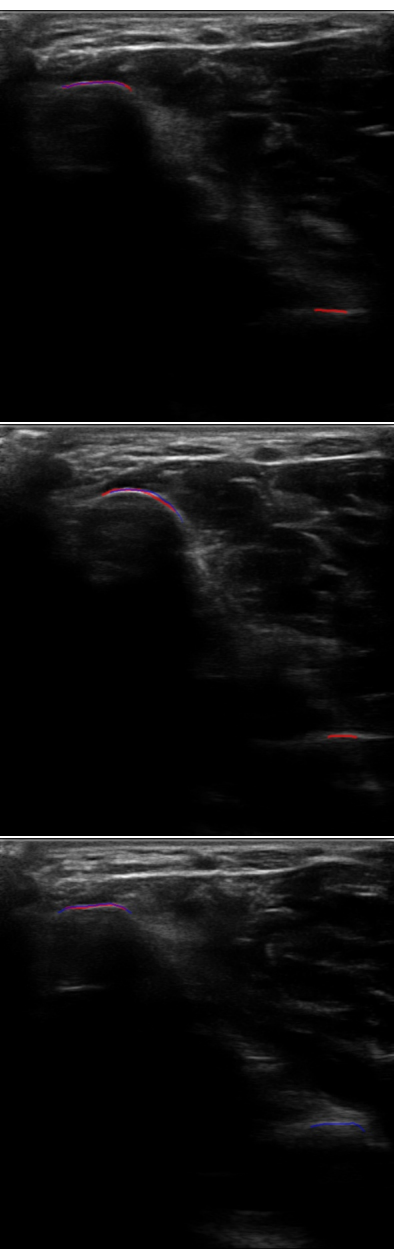

(c) oHD

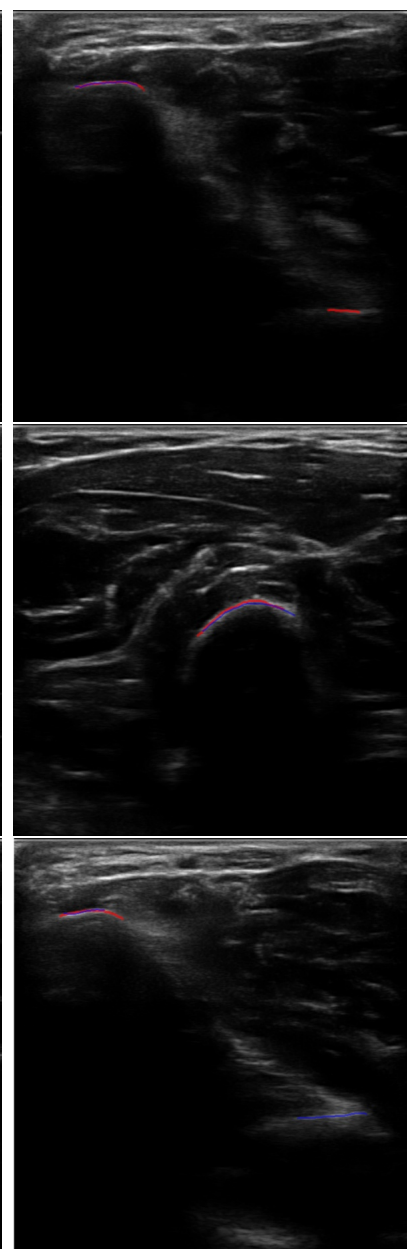

(d) $\mathrm{sHD}$

Figure 4: Qualitative evaluation of our proposed FCN(blurred) method. Red points show segmentations as performed by the network, blue points show the manual gold standard annotation. The top row shows images with minimum error (best-case) for the corresponding column evaluation metric, the middle row shows medianerror cases, and the bottom row shows the images for the maximum error (worst-case) for each corresponding metric. Network segmentations look better than those generated with U-Net (hairline) as shown in Figure 2 despite the higher quantitative error.

postprocessing of the neural network output requires one to know in advance how many bones are visible in a given US frame at test time.

In conclusion, we have shown that deep learning methods are well suited for bone surface segmentation in US. Our presented models only require a final thinning step to obtain a single-pixel thick delineation for applications in which such output is desired. In future work, we plan to extend our current dataset with US images acquired using different transducers and anatomies, and use the segmentation framework for fully automatized registration.

Acknowledgements. This work was funded by the Swiss National Science Foundation (SNSF) and a Highly Specialized Medicine (HSM2) grant of the Canton of Zurich. TensorFlow implementations are based on work by Christian Baumgartner. We thank NVIDIA for their GPU donations.

\section{REFERENCES}

1. M. Salehi, R. Prevost, J. L. Moctezuma, N. Navab, and W. Wein, "Precise ultrasound bone registration with learning-based segmentation and speed of sound calibration," in Medical Image Computing and Computer 


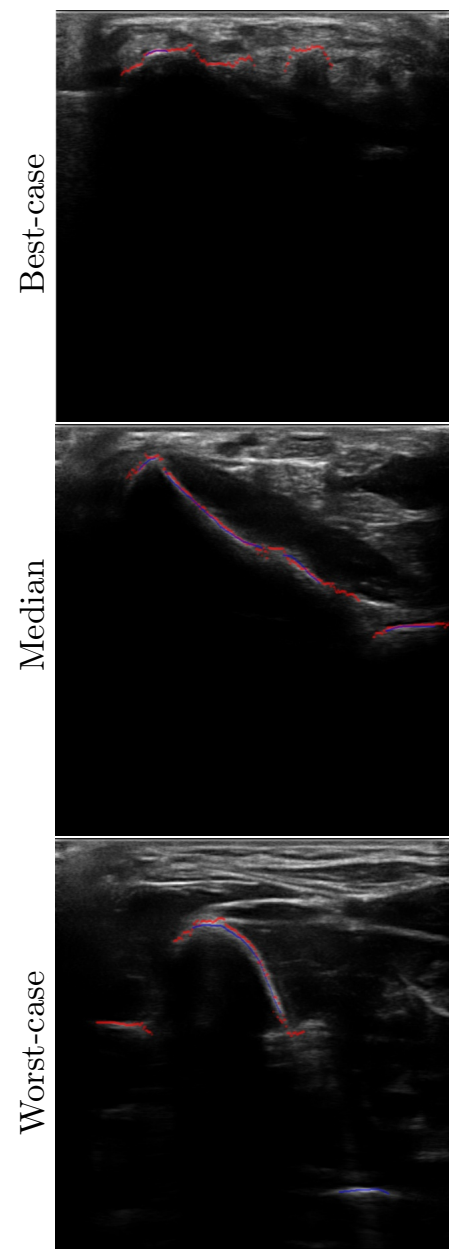

(a) RMSE

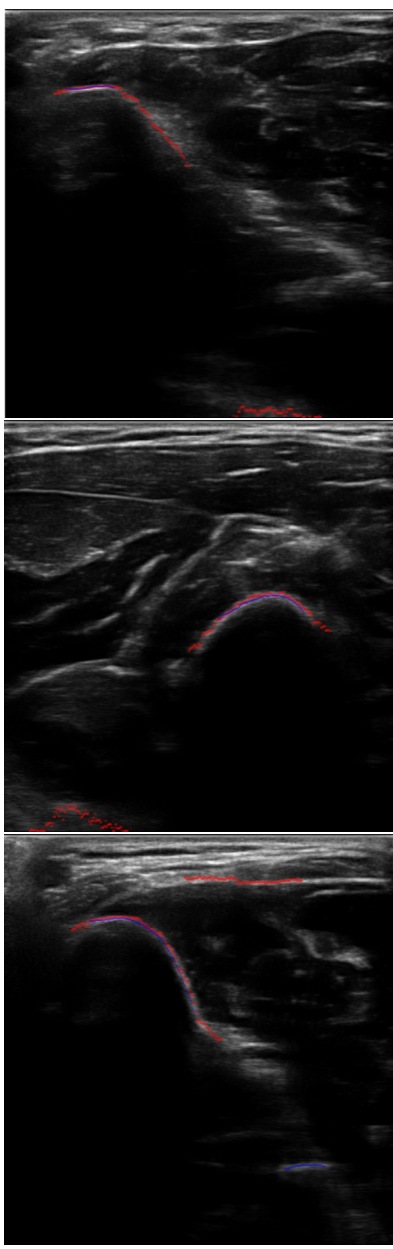

(b) SME

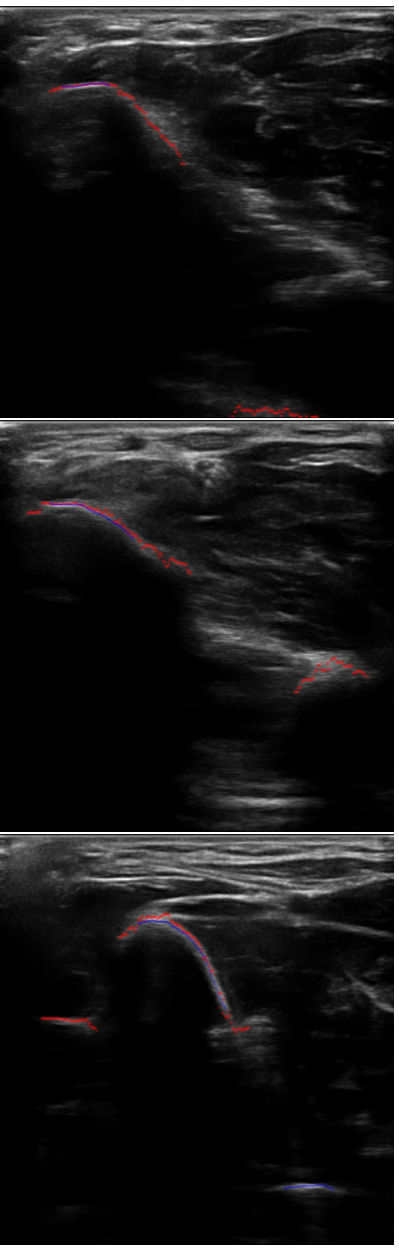

(c) oHD

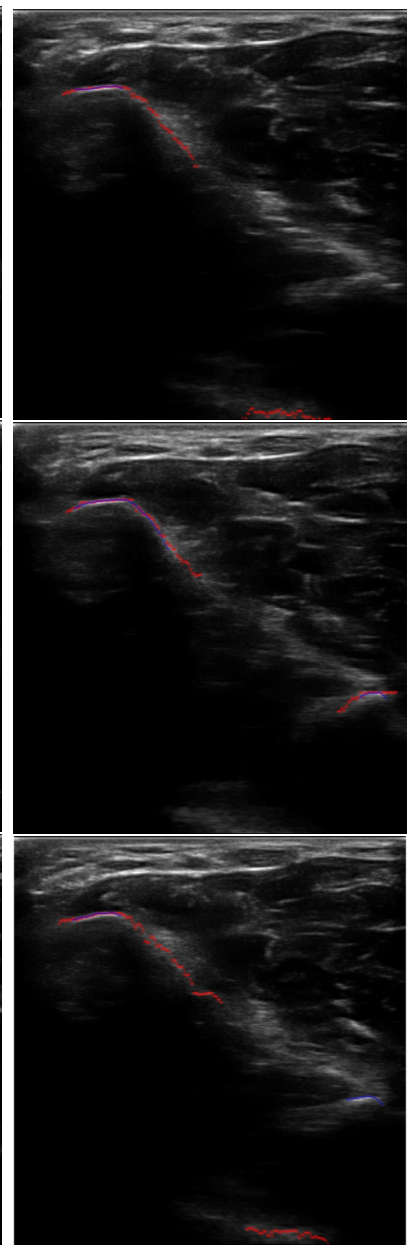

(d) $\mathrm{sHD}$

Figure 5: Qualitative evaluation of the baseline method. Red points show segmentations as performed by the network, blue points show manual gold standard. The top row shows images with minimum error (best-case) for the corresponding column evaluation metric, the middle row shows median-error cases, and the bottom row shows the images for the maximum error (worst-case) for each corresponding metric.

Assisted Intervention - MICCAI 2017 - 20th International Conference, Quebec City, QC, Canada, September 11-13, 2017, Proceedings, Part II, M. Descoteaux, L. Maier-Hein, A. Franz, P. Jannin, D. L. Collins, and S. Duchesne, eds., Lecture Notes in Computer Science 10434, pp. 682-690, Springer, 2017.

2. M. Ciganovic, F. Ozdemir, F. Pean, P. Fuernstahl, C. Tanner, and O. Goksel, "Registration of 3d freehand ultrasound to a bone model for orthopedic procedures of the forearm," International Journal of Computer Assisted Radiology and Surgery 13, pp. 827-836, Jun 2018.

3. I. Hacihaliloglu, A.Rasoulian, R.N.Rohling, and P. Abolmaesumi, "Local phase tensor features for 3-D ultrasound to statistical shape+pose spine model registration," IEEE TMI 33, pp. 2167-2179, 2014.

4. S. Khallaghi, C. A. Sánchez, J. Sun, F. Imani, A. K. G. Khale, O. Goksel, A. Rasoulian, C. Romagnoli, H. Abdi, S. Chang, P. Mousavi, A. Fenster, A. Ward, S. Fels, and P. Abolmaesumi, "Biomechanically constrained surface registration: Application to MR-TRUS fusion for prostate interventions," IEEE TMI 34(11), pp. 2404-14, 2015.

5. I. Hacihaliloglu, R. Abugharbieh, A. Hodgson, and R. N. Rohling, "Bone surface localization in ultrasound using image phase-based features," Ultrasound Med Biol 35(9), pp. 1475-1487, 2009.

6. P. Kovesi, "Image features from phase congruency," Videre: Journal of Computer Vision Research 1(3), pp. 1-26, 1999. 
7. M. Felsberg and U. Köthe, "Get: The connection between monogenic scale-space and gaussian derivatives," in Scale Space and PDE Methods in Computer Vision, R. Kimmel, N. A. Sochen, and J. Weickert, eds., pp. 192-203, Springer Berlin Heidelberg, (Berlin, Heidelberg), 2005.

8. A. Karamalis, W. Wein, T. Klein, and N. Navab, "Ultrasound confidence maps using random walks," Med Image Anal 16(6), pp. 1101-1112, 2012.

9. N. Quader, A. Hodgson, and R. Abugharbieh, "Confidence weighted local phase features for robust bone surface segmentation in ultrasound," pp. 76-83, 092014.

10. F. Ozdemir, E. Ozkan, and O. Goksel, "Graphical modeling of ultrasound propagation in tissue for automatic bone segmentation," in MICCAI, pp. 256-264, 2016.

11. N. Baka, S. Leenstra, and T. van Walsum, "Machine learning based bone segmentation in ultrasound," in Computational Methods and Clinical Applications for Spine Imaging, J. Yao, T. Vrtovec, G. Zheng, A. Frangi, B. Glocker, and S. Li, eds., pp. 16-25, Springer International Publishing, (Cham), 2016.

12. N. Baka, S. Leenstra, and T. van Walsum, "Ultrasound aided vertebral level localization for lumbar surgery," IEEE Transactions on Medical Imaging 36, pp. 2138-2147, Oct 2017.

13. O. Ronneberger, P. Fischer, and T. Brox, "U-net: Convolutional networks for biomedical image segmentation," in Medical Image Computing and Computer-Assisted Intervention - MICCAI, N. Navab, J. Hornegger, W. M. Wells, and A. F. Frangi, eds., pp. 234-241, Springer International Publishing, (Cham), 2015.

14. A. K. Jain and R. H. Taylor, "Understanding bone responses in b-mode ultrasound images and automatic bone surface extraction using a bayesian probabilistic framework," Proc.SPIE 5373, pp. 5373 - 5373 - 12, 2004 .

15. J. Long, E. Shelhamer, and T. Darrell, "Fully convolutional networks for semantic segmentation," CoRR abs/1411.4038, 2014.

16. S. Ioffe and C. Szegedy, "Batch normalization: Accelerating deep network training by reducing internal covariate shift," 2015.

17. D. P. Kingma and J. Ba, "Adam: A method for stochastic optimization," 2014. 